\title{
7 Die geschlossenen Venerologischen Stationen der Hautklinik des Krankenhauses Dresden-Friedrichstadt
}

Im Mai 1945 entschied die Stadtverwaltung von Dresden, Karl Linser die Leitung der Friedrichstädter Hautklinik zu übertragen (Kap. 3.4). Das Krankenhaus Dresden-Friedrichstadt, das am 27. November 1849 im sogenannten Palais des Grafen Marcolini eröffnet wurde, wurde im Zweiten Weltkrieg vergleichsweise wenig zerstört. ${ }^{543}$ Lediglich das Hauptgebäude, in dem unter anderem die Hautklinik untergebracht war, musste vor Kriegsende evakuiert werden, da es durch Brände beschädigt war. Bereits nach wenigen Monaten war die Hautklinik wieder soweit aufgebaut, dass 300 Patienten im Ostflügel des Marcolini-Palais aufgenommen werden konnten, dem neuen Sitz der Hautklinik. Hilfreich beim Wiederaufbau war auch die lange Tradition der Hautklinik bei der Beratung und Behandlung von Geschlechtskranken, die bis in die Zeit vor dem Ersten Weltkrieg reichte. So wurden seit den 1920er Jahren geschlechtskranke Männern und Frauen zugeführt und in den geschlossenen Abteilungen der Hautklinik des Krankenhauses Dresden-Friedrichstadt zwangsweise behandelt. ${ }^{544}$ In kurzer Zeit gelang es Karl Linser, die Hautklinik von DresdenFriedrichstadt als Zentrum für die Behandlung von Geschlechtskrankheiten

543 Kunze E (Hrsg.) (1999) Ein Palais erzählt. Das Marcolini-Palais und Friedrichstadt, ein Stück europäischer Geschichte. Krankenhaus Dresden-Friedrichstadt Dresden.

544 Hansel G (2005) Die Geschichte der Hautklinik Dresden Friedrichstadt. Med. Diss., Dresden, S. $38 \mathrm{ff}$. 
7 Die geschlossenen Venerologischen Stationen der Hautklinik des Krankenhauses Dresden-Friedrichstadt

in Dresden wieder zu etablieren. Nicht zuletzt vor diesem Hintergrund wurde er im Februar 1946 zum sächsischen Landesvenerologen ernannt. ${ }^{545}$

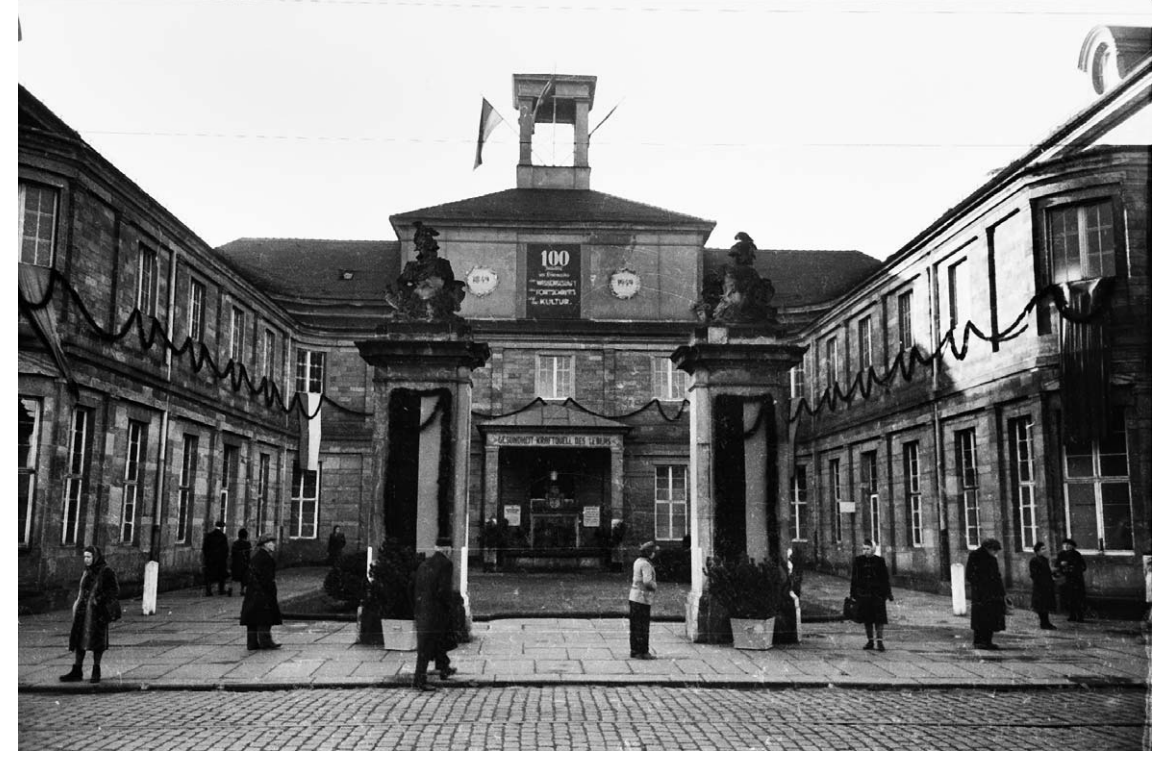

Abb. 20 Marcolini-Palais in Dresden-Friedrichstadt (um 1947)

1947 erhielt Karl Linser eine Professur an der Universitätshautklinik in Leipzig. ${ }^{546}$ Im selben Jahr musste auf Grundlage des SMAD-Befehls Nr. 30 im Krankenhaus Dresden-Friedrichstadt eine Poliklinik eingerichtet werden. Die hierfür benötigten Räume sollte die Hautklinik bereitstellen, sodass die venerologischen Patienten von Dresden-Friedrichstadt in das Infektionskrankenhaus Dresden-Taucha verlegt wurden. Zwei Jahre später, im April 1949, wurden die 80 Betten für venerologische Patienten erneut verlegt. Die neue Unterkunft war das Hilfskrankenhaus Bodelschwinghstraße, das ursprünglich 1928 als Obdachlosenasyl errichtet wurde und anschließend als Seuchenkrankenhaus diente. ${ }^{547}$ Hier wurden zwei geschlossene Venerologische Stationen eingerichtet - eine für geschlechtskranke Männer und eine für geschlechtskranke Frauen - wie aus einem Interview mit einem ehemaligen Arzt der Hautklinik Dresden-Friedrichstadt hervorgeht: „Nach dem Krieg, das Ganze ist ja nicht jetzt von der DDR, diese geschlossenen Abteilungen sind eigentlich schon bei der sowjetischen Militäradministration, und da gab es so einen Befehl und da gab

545 Kunze P (1999) Vom Adelspalais zum Städtischen Klinikum. Geschichte des Krankenhauses Dresden-Friedrichstadt, zusammengestellt anläßlich des 150jährigen Jubiläums 1849-1999. Krankenhaus Dresden-Friedrichstadt Dresden, S. 118.

546 Kunze: Vom Adelspalais zum Städtischen Klinikum (Anm. 545), S. 119.

547 Kunze: Vom Adelspalais zum Städtischen Klinikum (Anm. 545), S. 119. 
7 Die geschlossenen Venerologischen Stationen der Hautklinik des Krankenhauses Dresden-Friedrichstadt

es in Dresden eine venerologische Abteilung auf der Bodelschwinghstraße. " 548 Leiter der geschlossenen Venerologischen Abteilung in der Bodelschwinghstraße wurde Wenzel Loew ( $\left.{ }^{*} 1910\right)$, der seit 1948 in Dresden-Taucha die venerologische Frauenabteilung betreut hatte. Nach einem Bericht aus dem Jahr 1952 wurde im Hilfskrankenhaus Bodelschwinghstraße die Bettenzahl für venerologische Patienten auf 117 Betten erhöht, ${ }^{549}$ die auf die Station 1 (venerologische und hautkranke Männer), die Station 2 (hautkranke Frauen und spätsyphilitische) und die Station 3 (venerologische Frauen) verteilt wurden.

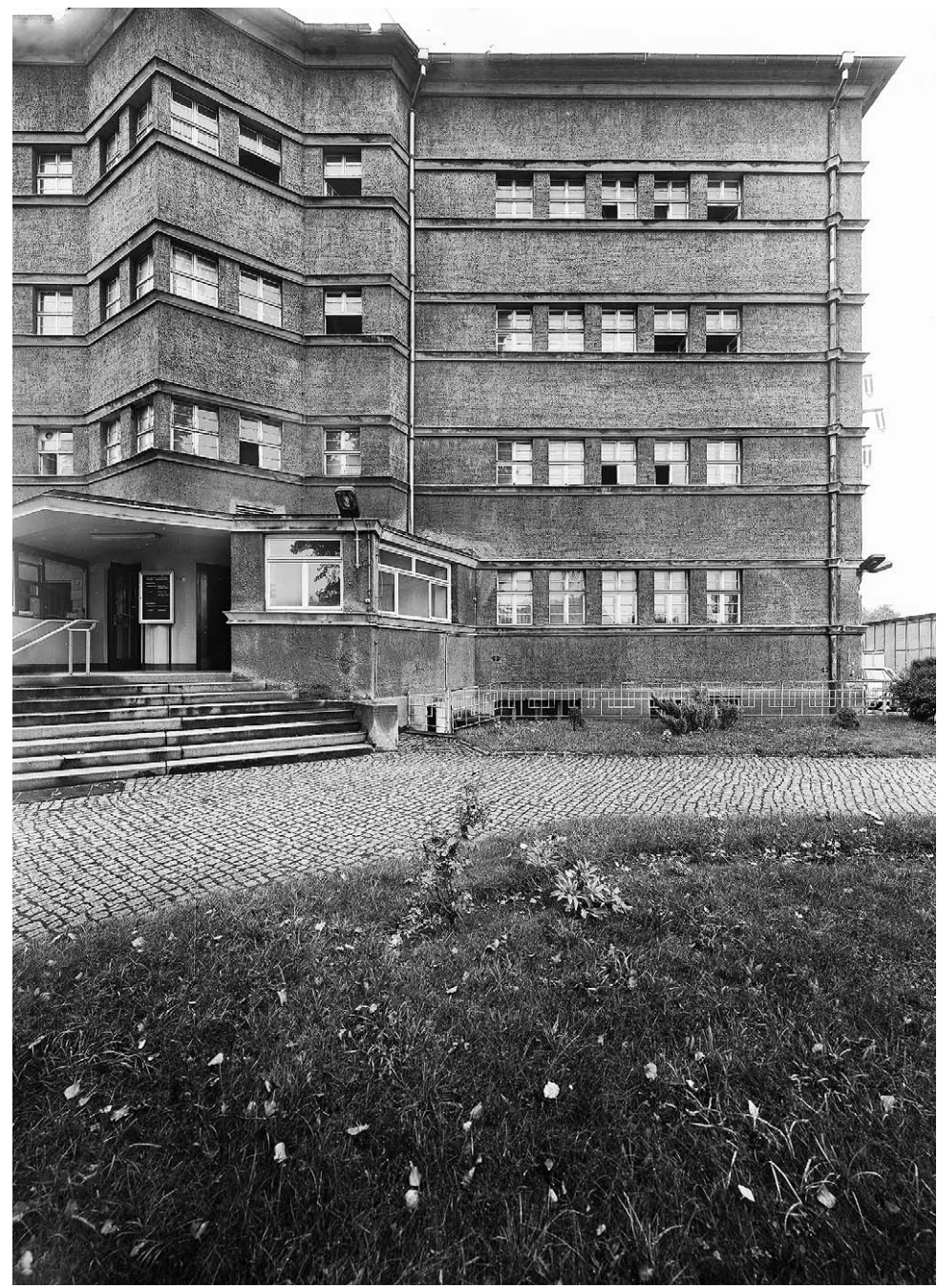

Abb. 21 Hilfskrankenhaus Bodelschwinghstraße in Dresden (um 1947)

548 Interview mit Herrn DAA (Anm. 307)

549 Kunze: Vom Adelspalais zum Städtischen Klinikum (Anm. 545), S. 119. 
7 Die geschlossenen Venerologischen Stationen der Hautklinik des Krankenhauses Dresden-Friedrichstadt

Nach dem Weggang von Karl Linser wurde die Hautklinik in Dresden-Friedrichstadt im raschen Wechsel erst von Joachim Bettermann ( $\left.{ }^{*} 1917\right)$ und ab August 1948 von Roderich Helmke (1906-1980) geleitet. Vor allem Helmke war mit der räumlichen Abtrennung der venerologischen Abteilung von der Hautklinik in Dresden-Friedrichstadt nicht einverstanden und beendete seine Tätigkeit 1949. $55^{\circ}$ Vorerst kommissarisch übernahm im Januar 1950 Heinz Hering die Leitung der Hautklinik und war damit auch für die geschlossenen Venerologischen Stationen in der Bodelschwinghstraße zuständig. Hering, der bis 1975 die gesamte Hautklinik (Marcolini-Palais und Bodelschwinghstraße) in Dresden-Friedrichstadt leitete, hatte nach dem Abitur in Dresden Humanmedizin in Jena, Breslau, Bonn und Leipzig studiert. ${ }^{551}$ Nach seinem Staatsexamen 1937 wurde er im November 1937 mit der Dissertationsschrift Experimentelle Beiträge zum Fettstoffwechsel der Psoriasis vulgaris552 zum Doktor der Medizin promoviert. Noch im selben Jahr wurde er Mitglied der NSDAP. 1942 folgte die Einberufung zur Wehrmacht und 1944 geriet er in englische Kriegsgefangenschaft, aus der er im Sommer 1945 entlassen wurde. 1944, möglicherweise während seiner Kriegsgefangenschaft, trat er aus der NSDAP aus. Im Oktober 1945 erhielt er die Zulassung als niedergelassener Arzt und eröffnete 1946 eine Praxis für Haut- und Geschlechtskrankheiten. ${ }^{553}$ Gleichzeitig übernahm er die Leitung des Hilfskrankenhauses Dresden-Leuben, wo er eine dermatovenerologische Sprechstunde einrichtete. Von 1950 bis 1974 war er Chefarzt der Hautklinik und Hautpoliklinik des Stadtkrankenhauses Dresden-Friedrichstadt. ${ }^{54}$ Anschließend übernahm er die Leitung der dermatologischen Abteilung in der Poliklinik Dresden-Niedersedlitz.

Die Aufteilung der Hautklinik in Dresden-Friedrichstadt im Marcolini-Palais einerseits und Bodelschwinghstraße andererseits bestand in der Amtszeit von Hering bis 1956.555 Bereits 1954 war aus dem Stadtkrankenhaus Dresden-Johannstadt die Medizinische Akademie Carl Gustav Carus hervorgegangen. ${ }^{556} 1957$ sollte im Verband der Medizinischen Akademie Carl Gustav Carus eine Hautklinik eingerichtet werden. In diesem Zusammenhang wurden 1957 Teile der Patienten sowie des medizinisch-pflegerischen Personals von der Hautklinik Dresden-Friedrichstadt an die Medizinische Akademie verlegt. Dabei handelte es sich um die venerologischen Patienten sowie die Ärzte und das Pflegepersonal aus dem Hilfskrankenhaus Bodelschwinghstraße. Im Gebäude der Bodelschwinghstraße wurde anschließend eine Krankenpflegeschule eingerichtet,

550 Kunze: Vom Adelspalais zum Städtischen Klinikum (Anm. 545), S. 119.

551 Hansel: Die Geschichte der Hautklinik Dresden Friedrichstadt (Anm. 544), S. 73.

552 Hering H (1937) Experimentelle Beiträge zum Fettstoffwechsel der Psoriasis vulgaris. Med. Diss., Leipzig.

553 Hansel: Die Geschichte der Hautklinik Dresden Friedrichstadt (Anm. 544), S. $75 f$.

554 Kunze: Vom Adelspalais zum Städtischen Klinikum (Anm. 545), S. 119.

555 Kunze: Vom Adelspalais zum Städtischen Klinikum (Anm. 545), S. 120.

556 Scholz A (2001) Nachkriegsjahre und Wiederaufbau. In: Scholz A, Heidel CP, Lienert M (Hrsg.) Vom Stadtkrankenhaus zum Universitätsklinikum. 100 Jahre Krankenhausgeschichte in Dresden. Böhlau Köln, Weimar, Wien, S. $143-167$. 
heute befindet sich dort die Medizinische Berufsfachschule am Krankenhaus Dresden-Friedrichstadt. ${ }^{55}$ Zwischen 1958 und 1959 wurde die Hautklinik Dresden-Friedrichstadt umgebaut. Nach den Umbauarbeiten standen der Hautklinik im Marcolini-Palais 150 Betten für dermatologische Patienten und 30 Betten für venerologische Patienten zur Verfügung. Für venerologische Patienten richtete man zunächst die geschlossene Station 19 in einem Pavillon ein und 1961 die Station 9 in der dritten Etage des Marcolini-Palais. „Die in der dritten Etage der Klinik gelegene Station wurde im Sinne der neuen Verordnung zur Verhütung und Bekämpfung von Geschlechtskrankheiten vom 23. Februar 1961 als sogenannte ,geschlossene Station' für die Aufnahme von 15 venerologischen Patientinnen hergerichtet." $55^{8}$ Seit 1961 bestand also die Hautklinik aus vier Stationen. Im Erdgeschoss war die Station 8 (Erwachsene und Kinder), im ersten Stock war die Station 7 (Frauen), im zweiten Stock war die Station 6 (Männer) und schließlich im dritten Stock die Station 9 für venerologische Frauen. Wie aus einer Übersicht zur „Stationsaufteilung und Stationsführung“ hervorgeht, wurde die Station 9 von Käthe Kleeberg geführt. ${ }^{559}$

Dass Heinz Hering die Zwangseingewiesenen nicht nur medizinisch versorgte, sondern sie möglicherweise in Studien eingeschlossen hatte, verdeutlicht sein Beitrag Aktuelle Fragen auf dem Gebiet der Gonorrhoe aus dem Jahr 1957. Darin berichtete Hering von seinen Erfahrungen im Umgang mit Gonorrhoe-Patientinnen in der Hautklinik Dresden-Friedrichstadt. Zunächst kommt er zu der Einschätzung, dass „als Hauptmotive der hwG-Personen (...) Schwachsinn, Triebhaftigkeit, Haltlosigkeit und an letzter Stelle gewerbsmäßige Unzucht zu nennen" sind. Diese Personen durch Erziehung von ihrem Handeln abzuhalten, hielt Hering für aussichtslos. Weiter formuliert er: „Soweit es sich noch um jugendliche Personen handelt, können Erziehungsmaßnahmen, die insbesondere die Erlernung eines das Leben erfüllenden Berufes zum Ziel haben und die dem Geisteszustand des Betreffenden angepaßt sind, noch Erfolge bringen. Mit zunehmendem Alter ist damit nichts mehr zu erreichen. Die Unterbringung in Arbeitshäusern, Fürsorgeheimen usw., die auf gerichtlichen Beschluß erfolgten, zeigten ungenügende Resultate. Besonders ungünstig stellte sich, ähnlich der Erfahrungen bei der Hospitalisierung, heraus, daß sich dort haltlose Personen erst recht kennenlernten und der negative Einfluß sich schneller als ein positiver durchsetzte. Hier müßte auf Grund gesetzgeberischer Maßnahmen versucht werden, die Betroffenen in kleinen Gruppen möglichst in Landgebieten unterzubringen, wo ein Untertauchen wie in Großstädten oder in Großbetrieben unmöglich ist und wo unter geeigneter Anleitung eine nützliche Arbeit, die auch den Lebensunterhalt sichert, zugewiesen werden kann. "560

557 Kunze: Vom Adelspalais zum Städtischen Klinikum (Anm. 545), S. $120 f$.

558 Hansel: Die Geschichte der Hautklinik Dresden Friedrichstadt (Anm. 544), S. $65 f$.

559 Kunze: Vom Adelspalais zum Städtischen Klinikum (Anm. 545), S. 121; Hansel: Die Geschichte der Hautklinik Dresden Friedrichstadt (Anm. 544), S. 66.

560 Hering: Aktuelle Fragen auf dem Gebiet der Gonorrhoe (Anm. 114), S. 182. 
7 Die geschlossenen Venerologischen Stationen der Hautklinik des Krankenhauses Dresden-Friedrichstadt

Da der Gesetzgeber aber nicht handelte und keine Landverschickung mit anschließender Isolierung vorsah, müsse zumindest die medizinische Versorgung besser geregelt werden, so Hering. Dazu gehöre nach Hering die genaue Untersuchung der Patienten, eine abgesicherte Diagnose und die Vermeidung von Rezidiven. Vor allem die Frage der Rezidive stand im Vordergrund der vorgestellten Studie, die Hering mit Patienten der Hautklinik Dresden-Friedrichstadt zwischen 1951 und 1955 durchgeführt hatte. Ein Ergebnis dieser Studie ergab „die eindeutige Forderung, bei hwG-Personen und hospitalisierungspflichtigen Go[norrhoe]-Kranken mindestens bis zum 2o. Präparat stationär zu untersuchen und auch ambulant die Untersuchung über genügend lange Zeit auszudehnen, wobei die Zeit wichtiger ist als die Anzahl der Abstriche. "561 Mit seiner Forderung ging Hering klar gegen Überlegungen zu einer Liberalisierung im Umgang mit Gonorrhoe-Patienten vor, was auch in seiner Haltung gegenüber einer Aufhebung der stationären Versorgung deutlich wurde: „Oskar Gans schlug 1950 vor, sogar auf die stationäre Behandlung von hwG-Personen, die mit Go[norrhoe] behaftet waren, zu verzichten, was ich aber allein schon aus Gründen der Gefahr des Wegfalls jeder Hemmung und der damit gegebenen Erhöhung der Promiskuität ablehnen möchte. “562 Ob Heinz Hering für diese Studie ausschließlich auf männliche und weibliche Patienten der geschlossenen Abteilungen der Bodelschwinghstraße zurückgegriffen hatte, geht aus seinem Beitrag nicht hervor.

Die durch Heinz Hering im Jahr 1961 eingerichtete geschlossene Venerologische Station im Marcolini-Palais hatte zumindest bis 1974 Bestand, dem Jahr, in dem Hering die Hautklinik verließ. Der Umgang mit zwangseingewiesenen Geschlechtskranken gehörte zur ärztlichen Routine von Hering. Bereits in der „Vorsulfonamid- und Vorpenizillinära“, wie Hering es umschreibt, wurden an der Hautklinik Dresden-Friedrichstadt „Insassinnen der geschlossenen Station" mit Gonorrhoe behandelt. ${ }^{56} \mathrm{Als}$ leitender Facharzt des dermatologischen Hilfskrankenhauses Dresden-Leuben war er mit der Behandlung von Geschlechtskrankheiten sowie den Zwangshospitalisierungen vertraut - die Leiter der Ambulatorien und Hilfskrankenhäuser waren nach SMAD-Befehl Nr. 30 zur namentlichen Meldung und Infektionsquellenforschung verpflichtet. Zudem war Hering nach der Berufung von Karl Linser an die Hautklinik der Universität Leipzig über mehrere Jahre Bezirksbeauftragter zur Bekämpfung der Geschlechtskrankheiten in Dresden. In dieser Funktion mussten die Zwangseinweisungen von ihm abgezeichnet werden. Schließlich war er als Chefarzt der Hautklinik Dresden-Friedrichstadt mit der Behandlung der Zwangseingewiesenen erst in der Abteilung Bodelschwinghstraße und später in der geschlossenen Venerologischen Station im Marcolini-Palais betraut.

561 Hering: Aktuelle Fragen auf dem Gebiet der Gonorrhoe (Anm. 114), S. 187.

562 Hering: Aktuelle Fragen auf dem Gebiet der Gonorrhoe (Anm. 114), S. 186.

563 Hering: Aktuelle Fragen auf dem Gebiet der Gonorrhoe (Anm. 114), S. 185. 


\subsection{Altag auf der Station}

Im Februar 1974 erhielt eine Zeitzeugin, die in der geschlossenen Venerologischen Station der Hautklinik des Krankenhauses Dresden-Friedrichstadt zwangseingewiesen wurde, Post von der Beratungs- und Fürsorgestelle Dresden: „Und dann habe ich Post bekommen, ich sollte mich in Friedrichstadt melden aufgrund eines Vorfalls oder so, kann ich nicht mehr so sagen und zu dem Zeitpunkt sollte ich früh um sechs meine Tochter runterbringen an die Haustüre und die käme dann zur Kur mit dem Transport, was eigentlich ungewöhnlich war. Wirklich ungewöhnlich, aber ich hatte kein schlechtes Gewissen, auch nichts dabei gedacht. Die ist zur Kur und (...), wenn ich nicht komme, dann würde ich geholt mit der Polizei, ja und da bin ich hin, sollte Nachtwäsche und alles mitbringen. Da bin ich hin, das war ganz oben auf der Station 9 und wie ich dort rein bin, ging hinter mir die Tür zu, wurde zugeschlossen und dann ging der Terror los. "Vor der Einweisung in Dresden-Friedrichstadt hatte die Zeitzeugin keine weiteren Aufforderungen erhalten, sich bei einem Hautarzt oder Gynäkologen vorzustellen. Es gab weder freiwillige noch angewiesene Untersuchungen im Voraus. „Nein, nur ich sollte zu dem Zeitpunkt auf der Station erscheinen und vorher nichts und gar nichts. "Warum die Zeitzeugin in die geschlossene Venerologische Station eingewiesen wurde, erklärt sie sich heute wie folgt: „Das kann ich Ihnen genau sagen. Also ich war schon immer ein Gegner mit dem Regime, habe das auch kundgetan. Dann hatte mein Bruder eine Ausreise, meine Schwester eine Ausreise; (...) die haben das so begründet, das war wie so ein Denkzettel, also wenn du jetzt nicht langsam mal ein bisschen ruhig bist oder was, das ist erst einmal ein Vorgeschmack und dann kann Schlimmeres passieren. "564

Zur Station 9 im dritten Stock der Hautklinik Dresden-Friedrichstadt ging eine „Treppe hoch, Fahrstuhl gab es, glaube ich, damals gar nicht. Dann habe ich aber schon diese Türe gesehen, diese Gittertüre und habe mir aber nichts dabei gedacht. “ Bereits bei der Aufnahme in die Station wurde der Zeitzeugin gesagt, dass sie „dableiben muss, bis die ganzen Untersuchungen abgeschlossen sind“. Auch dass die Station verschlossen werden würde, „wurde uns gesagt, dass wir nicht abhauen, obwohl das auf der Station gar nicht möglich war. Nach zwei Gittertüren war man ja schon da. "Auf den ersten Blick war die geschlossene Venerologische Station g eine ganz normale Station, „aber die Türen wurden zugeschlossen und dann waren zwischendurch immer solche großen Gittertüren, dann ging es die Treppen runter, war auch noch mal eine Türe, eine Gittertüre, ja und die Toiletten waren draußen. Mussten pochen, wenn wir raus wollten. Nachts wurde generell zugeschlossen und ich war mit einer FDJ-[Freie Deutsche Jugend]-Sekretärin zusammen im Zimmer und weil ich Platzangst kriegte, die habe ich bis heute noch, bestimmt dadurch, die

564 Interview mit Frau DPA, geführt von Florian Steger und Maximilian Schochow, Halle (Saale), den 4. November 2014. 
7 Die geschlossenen Venerologischen Stationen der Hautklinik des Krankenhauses Dresden-Friedrichstadt

sagte zu mir, ich bin ja immer Nichtraucher gewesen, ich bin mal hier operiert worden wegen ein bisschen tiefer Stimme, sie sagte: ,Ich gebe dir eine Zigarette, damit du mal rauskommst, dann gehst du einfach mit in den Raucherraum. 'So haben wir das dann gehandhabt." Die Station hatte mehrere Zimmer: „Das waren viele Zimmer, sieben, acht bestimmt.“ Da auf der Station 15 Betten für venerologische Frauen vorgehalten wurden, dürfte die Station aus Zweibettzimmern bestanden haben, in denen zwei Zwangseingewiesene untergebracht waren: „Zwei, ich war mit der FDJ-Sekretärin zusammen. “ Die Fenster „waren verschlossen und Gitter dran“. ${ }^{565}$

Bei der Aufnahme auf die Station musste die Zwangseingewiesene ihre Kleidung ausziehen und zur Verwahrung abgeben - „wir mussten Sachen ausziehen. Wir hatten solche Anzüge angehabt." Anschließend erfolgte eine gynäkologische Untersuchung, die dann zur täglichen Routine wurde. „Da gab es Ärzte, Schwestern kaum, Ärzte und wir mussten jeden Früh und alle (...) auf den Stuhl so drauf. Jeden Tag untersucht, jeden Tag, sinnloserweise." Das Vorgehen des behandelnden Arztes wird als grob beschrieben: „Na zu uns allen brutal. (...) Also rigoros und unsensibel." "566 Wie auch in Bezug auf die Abteilung in Berlin-Buch beschrieben, herrschte auf der geschlossenen Station Langeweile. „Wir saßen im Zimmer, haben uns unterhalten und wie gesagt, mit mir die Frau, die ging öfters mal rauchen, bis sie dann gesagt hat: ,Dann komm doch mit, ich gebe dir eine Zigarette und du tust einfach so. ““567 Arbeiten oder Hilfstätigkeiten, wie für andere geschlossene Venerologische Stationen beschrieben, wurden nicht verrichtet. Demnach bestand der Zweck der geschlossenen Venerologischen Station der Hautklinik des Krankenhauses Dresden-Friedrichstadt in der Behandlung möglicher Krankheiten und im Verwahren und Wegsperren. Gewalttätige Übergriffe durch die Zwangseingewiesenen selbst, wie in Halle (Saale) oder Berlin-Buch, sind für Dresden-Friedrichstadt nicht belegt: „Nein, nein. (...) Wir waren alle bedrückt und irgendwie schockiert. Die wurden ja alle aus ihren Familien herausgerissen. Das war ja nicht, du kommst übermorgen, das war ja praktisch von heute auf morgen, ja. " ${ }^{668}$ Im Gegenteil sprechen die Beschreibungen des Verhältnisses zwischen der Zeitzeugin und ihrer Bettnachbarin für ein gutes Miteinander der Zwangseingewiesenen. Möglicherweise vereinte die beiden Frauen ihre politische Haltung, denn die FDJ-Sekretärin war wahrscheinlich auch aus politischen Gründen in der geschlossenen Venerologischen Station der Hautklinik des Krankenhauses Dresden-Friedrichstadt: „Das kann dann politisch gewesen sein. Die hat mir ja erzählt, dass sie auch gegen den Staat agiert hat. " ${ }^{569}$ 
Zu ihrer Tochter, die zu diesem Zeitpunkt zur Kur in Graal-Müritz war, durfte die Zeitzeugin keinen Kontakt aufnehmen. „Also ich war dann eine Zeit ziemlich unruhig, muss ich mal sagen. Ich hatte Angst. Es ging auch um mein Kind. Ich habe ja die ganze Zeit nichts erfahren, was mit ihr ist. (...) Ich habe immer nachgefragt, können Sie nicht mal dort anrufen, die war in Graal-Müritz zur Kur, die war noch so klein, wie es ihr geht oder was, nichts, gar nichts. " 570 Auch zu anderen Personen durfte kein Kontakt aufgenommen werden. „Na da durfte ja niemand hin. Ich habe ja auch niemanden sagen dürfen, wo ich hingehe. Ich bin dort hin und habe auch niemandem Bescheid gesagt." Bei der Entlassung wurde der Zeitzeugin mitgeteilt, dass ein Krankheitsverdacht vorgelegen habe, „was sich dann aber herausgestellt hat, als ich entlassen worden bin, dass es nicht so gewesen ist“". ${ }^{771}$ Wie viele Zwangseingewiesene wurde auch diese Zeitzeugin vier Wochen ohne jede Diagnose eingesperrt.

\subsection{Die Auflösung der Station}

Nachdem Heinz Hering seine Chefarzttätigkeit an der Hautklinik DresdenFriedrichstadt beendete, wurde die Klinik ab 1974 kommissarisch durch Gerhard Goßrau (*1934) geleitet. ${ }^{572}$ Goßrau war erster Oberarzt bei Hering und hatte sich seit den 196oer Jahren auf die dermatologische Strahlentherapie spezialisiert. ${ }^{573}$ Seine kommissarische Leitung währte bis 1976, dem Jahr, das in einem doppelten Sinn einen Neuanfang der Hautklinik des Krankenhauses Dresden-Friedrichstadt symbolisiert. Zum einen wurde am 19. April 1976 Dr. med. Claus Seebacher $\left({ }^{*} 1935\right)$ Chefarzt der Hautklinik. Zum anderen wurde unter seiner Leitung das marode Gebäude der Hautklinik saniert. ${ }^{574}$ In der Folge dieser Sanierung wurde unter anderem die Station 9 umgestaltet und die geschlossene Venerologische Station aufgelöst. Beide Aspekte stehen für eine Modernisierung sowohl des Gebäudes als auch der Behandlung der Patienten.

Claus Seebacher hatte in Doberlug-Kirchhain 1954 sein Abitur gemacht und nahm im selben Jahr in Greifswald das Studium der Humanmedizin auf. 1955 wechselte er nach Leipzig und wenige Zeit später an die Medizinische Akademie Carl Gustav Carus in Dresden. ${ }^{775}$ Nach seinem Staatsexamen 1959 wurde er 1960 mit der Dissertationsschrift Strahlenbehandlungsergebnisse bei malignen Hodengeschwülsten in den letzten 30 Jahren, aufgezeigt am Krankengut der Strahlenklinik der Medizinischen Akademie Dresden ${ }^{576}$ promoviert. Für sein allgemeinärztliches

570 Interview mit Frau DPA (Anm. 564).

571 Interview mit Frau DPA (Anm. 564).

572 Scholz: Geschichte der Dermatologie in Deutschland (Anm. 38), S. 212.

573 Hansel: Die Geschichte der Hautklinik Dresden Friedrichstadt (Anm. 544), S. 90.

574 Vgl. hierzu ausführlich Hansel: Die Geschichte der Hautklinik Dresden Friedrichstadt (Anm. 544), S. 85-89.

575 Köstler E (2000) Professor Dr. Claus Seebacher zum 65. Geburtstag. Der Hautarzt 51, S. 791-792, hier: S. 791.

576 Seebacher C (1960) Strahlenbehandlungsergebnisse bei malignen Hodengeschwülsten in den letzten 30 Jahren, aufgezeigt am Krankengut der Strahlenklinik der Medizinischen Akademie Dresden. Med. Diss., Dresden. 
7 Die geschlossenen Venerologischen Stationen der Hautklinik des Krankenhauses Dresden-Friedrichstadt

Jahr ging er 1961 ans Kreiskrankenhaus Freital. Anschließend wechselte er für seine Facharztausbildung an die Dresdner Akademie-Hautklinik, die 1957 von Dr. med. Heinz Egon Kleine-Natrop (1917-1985) in Dresden Johannstadt als Teil der Medizinischen Akademie gegründet wurde. ${ }^{577}$ Dort erhielt Seebacher 1965 seine Facharztanerkennung und war bis 1971 als Assistenzarzt tätig. 1977 habilitierte er sich mit der Arbeit Epidemiologischer und experimenteller Beitrag zur Pathogenese der Dermatitis seborrhoides infantum. ${ }^{578}$ Seit Anfang der 196oer Jahre hatte sich Seebacher auf Mykologie spezialisiert, wurde 1972 in den Vorstand der Gesellschaft für Medizinische Mykologie der DDR gewählt und war ab 1975 Sekretär dieser Gesellschaft. ${ }^{579}$ Vier Jahre später wurde Seebacher zum Honorardozenten und 1985 zum Honorarprofessor für Dermatologie und Venerologie der Akademie für Ärztliche Fortbildung der DDR berufen. ${ }^{580}$

Mitte der 1970er Jahre wurde die Station 9 noch für die Unterbringung von zwangseingewiesenen Frauen genutzt, wie aus den Aussagen eines Arztes der Hautklinik hervorgeht. „Ja, ja, als ich dahin kam, war die Station noch zugeschlossen, das ist richtig. " ${ }^{81}$ Auch 1976, als Claus Seebacher die Hautklinik übernahm, war die Station noch verschlossen. Ob die geschlossene Station unmittelbar mit der Amtsübernahme von Seebacher aufgelöst wurde, bleibt ungewiss: „Also, ob da mal ein Pfleger ein Zimmer zugeschlossen hätte, das kann ich Ihnen jetzt nicht mehr sagen, also das ist mir jetzt nicht mehr bewußt.“ Dem Zeitzeugen zufolge hatte sich die geschlossene Venerologische Station noch Ende der 1970er Jahre „von selber aufgelöst“. Zumindest in dieser Übergangsphase war die Station situationsbedingt entweder geschlossen oder offen: „Naja, nach meinem Kenntnisstand war die Station, also wir hatten einen Schlüssel, um da rein- und rauszugehen. Die Schwester konnte mit dem elektrischen Türöffner die Tür öffnen. (...) So war es. Wer sich vernünftig benommen hat, konnte rausgehen, konnte in den Park gehen und so weiter. Wenn jetzt irgendwelche völlig renitent waren, wiederholt von der Polizei zugeführt worden sind, weiß ich gar nicht, was da passiert ist." Ceschlossen untergebracht wurden demnach vor allem jene Patienten, „die jetzt nicht willig waren, die wurden zugeführt“. Die meisten blieben dann auch freiwillig - „meistens ja." Es gab aber auch Patientinnen, die versuchten auszubrechen. Einige schienen es auch geschafft zu haben, „dann wurden sie wieder zugeführt“. ${ }^{882}$

Der Arzt erklärt sich, warum die geschlossene Venerologische Station der Hautklinik Dresden-Friedrichstadt eingerichtet und Ende der 197oer Jahre aufgelöst wurde, mit zwei Punkten. Die Einrichtung begründet er mit einem histori-

577 Scholz: Geschichte der Dermatologie in Deutschland (Anm. 38), S. 142.

578 Seebacher C (1976) Epidemiologischer und experimenteller Beitrag zur Pathogenese der Dermatitis seborrhoides infantum. Med. Diss., Dresden.

579 Köstler: Professor Dr. Claus Seebacher zum 65. Geburtstag (Anm. 575), S. 791.

580 Hansel: Die Geschichte der Hautklinik Dresden Friedrichstadt (Anm. 544), S. 91.

581 Interview mit Herrn DAA (Anm. 307).

582 Interview mit Herrn DAA (Anm. 307). 
schen Argument: In „den sechziger Jahren war das kein Problem. Da wurden auch auf Kinderstationen die Türen zugeschlossen und so weiter, also da würde ich sehr zurückhaltend sein. Wissen Sie, die ganze Einstellung, ich meine gerade, was die Geschlechtskrankheiten anbelangt, das geht ja, von achtzehnhundert paar in siebziger Jahren wurden die Geschlechtskranken separiert und anders betrachtet. Und 1925 ist ja in Deutschland, also im gesamten Deutschen Reich auch eine extra Verordnung zur Behandlung und Bekämpfung der Geschlechtskrankheiten vom Parlament beschlossen worden. Das ist von den Nazis dann übernommen worden. Zu DDR-Zeiten, also dann von der russischen Militärverwaltung nochmal extra betont und herausgearbeitet worden und dann ist von der DDR das einundsechziger Gesetz entstanden ja. Das ist ja eine Chronologie und die geschlossenen Abteilungen. "Aufgelöst wurde die Station, weil sie für die sowjetische Militäradministration nicht mehr notwendig war, so der Zeitzeuge: „Das ist mir nicht bekannt, dass die in irgendeiner Form von DDR-Einrichtungen, also von der DDR eingerichtet sind. Das sind noch Relikte aus der Zeit vor Gründung der DDR, also als wir direkt unter sowjetischer Militärverwaltung sind. (...) Ich meine, die Geschlechtskrankheiten waren eigentlich immer schon eine besondere Sorte und die Militärs hatten einen Horror, wenn bei ihnen ganze Armeen den Tripper hatten können. " ${ }^{583}$ Demnach waren die geschlossenen Venerologischen Stationen nicht nur ein Relikt der Nachkriegszeit, sondern vor allem eine Maßnahme der SMAD und des Militärs. Dieser Kontext ist insofern bemerkenswert, als dass bis in die 196oer Jahre hinein, unter anderem von Heinz Hering, die geschlossenen Stationen als Verwahr- und Erziehungsanstalten für unbequeme Personen betrachtet wurden. ${ }^{84}$

Auch den Erziehungsauftrag, der in der Hausordnung der geschlossenen Venerologischen Station von Halle (Saale) sowie in der täglichen Disziplinierung in den geschlossenen Venerologischen Stationen von Berlin-Buch, Halle (Saale) oder Leipzig-Thonberg deutlich wird, interpretiert der Arzt im Kontext der sowjetischen Militäradministration: „Ja das steht ja in der russischen Verordnung, also in der sowjetischen da explizit drin. (...) Das sie also zur und was da, ich weiß jetzt nicht, wie es im DDR-Gesetz formuliert ist, das ist möglich. “ Einen Erziehungsauftrag für die Station in Dresden-Friedrichstadt habe es nicht gegeben „und letztendlich hätte man ja dazu auch dann entsprechend Personal gebraucht“. Dennoch kam es auch Ende der 197oer Jahre noch zu Disziplinierungsmaßnahmen, zumindest aber zur Androhung solcher Maßnahmen: „Da wurde dann zwar von dem alten Pfleger, wir hatten noch einen, der kam noch aus Gottron-Zeiten, aus Breslau und der kannte die ganzen Sachen. Der wusste also immer, wie die Gonorrhoe behandelt wurde, bevor das Penicillin eingeführt wurde. Dass der dann mal gesagt hat: ,Also, wenn ihr jetzt nicht spurt, dann gibt es eine Betonbombe.' oder ich weiß jetzt nicht mehr,

583 Interview mit Herrn DAA (Anm. 307).

584 Hering: Aktuelle Fragen auf dem Gebiet der Gonorrhoe (Anm. 114), S. 182. 
7 Die geschlossenen Venerologischen Stationen der Hautklinik des Krankenhauses Dresden-Friedrichstadt

wie der Ausdruck war. Das will ich jetzt gar nicht in Abrede stellen, dass wir jetzt als disziplinarische Maßnahmen verordnet hätten, denn das wäre ja eine ärztliche Anordnung. “" ${ }^{85}$ Mögliche Grenzüberschreitungen vonseiten der Patientinnen wurden also sanktioniert.

Unter der Leitung von Claus Seebacher wurde aber nicht nur die geschlossene Venerologische Station aufgelöst, sondern auch der Umgang mit den venerologischen Patientinnen geändert. Die geschlossene Venerologische Station wurde als offene Station weitergeführt. Auch eine durchschnittliche Behandlungszeit von acht Wochen, wie für Halle (Saale) dokumentiert, war Ende der 197oer Jahre in der Hautklinik Dresden nicht üblich. „Die durchschnittliche Liegezeit, also kann ich Ihnen jetzt nicht sagen, das wurde ja alles genau dokumentiert und auch berichtet. (...) Also fünf, sechs Wochen verstehe ich nicht. Es möge Einzelfälle geben, wo immer wieder zwischendurch mal ein Abstrich dabei ist, wo Zweifel sind, wo man nicht die Möglichkeiten, also in den sechziger Jahren, Anfang der siebziger Jahre, die Gonokokken zu kultivieren. Das war ausgesprochen schwierig. Die sind so anspruchsvoll und wir hatten in der DDR die entsprechenden Nährmedien nicht zur Verfügung, um die Kulturen, jetzt routinemäßig, durchführen zu können. PCR gab es eh nicht und andere Möglichkeiten eben auch nicht. Serologisch war das auch nicht machbar, sodass es schon mal in ganz seltenen Einzelfällen schwierig sein konnte, eine klare Diagnose rein morphologisch, mikromorphologisch zu stellen. “586

Spätestens in den 1980er Jahren wurde die Station 9 als offene und vor allem gemischte Station geführt. „Da war ja auf dieser Station, das war ja eine gemischte Station, da hatten wir so zwei oder drei Zimmer, je nachdem, wie viele. Planmäßig waren wohl so 15 Betten vorgesehen, aber die waren eigentlich selten, zumindestens zu meiner Zeit, belegt mit venerologischen Patientinnen, und diese Station war integriert in eine allgemeine Frauenstation. “ Für die stationäre Aufnahme mussten die venerologischen Patientinnen bestimmte Bedingungen erfüllen: „Stationär erging nur dann, wenn irgendwelche Probleme bestanden, also bei Komplikationen, aber auch, wenn disziplinarische Probleme waren, denn die Geschlechtskrankheiten, da bestand eine Behandlungspflicht und die Pflicht oder das Nichteinhalten dieser Gesetze konnte bestraft werden, ja." ${ }^{587}$

Dennoch wurden auch bei den stationär aufgenommenen venerologischen Frauen die Standardmethoden angewandt, wie sie in Gertlers Publikation Systematische Dermatologie und Grenzgebiete ${ }^{58}$ beschrieben werden (Kap. 4.5). Bei der chronischen Gonorrhoe beispielsweise, „die hintere, also wenn dann zum Beispiel bei den Frauen die Annexen befallen waren oder beim Mann vielleicht

585 Interview mit Herrn DAA (Anm. 307).

586 Interview mit Herrn DAA (Anm. 307).

587 Interview mit Herrn DAA (Anm. 307).

588 Gertler: Systematische Dermatologie und Grenzgebiete (Anm. 20), S. 1246. 
Prostata, Prostatitis und so weiter, Nebenhodenentzündung, das war dann schwieriger zu diagnostizieren, weil dann die Gonokokken nicht mehr in den vorderen Abschnitten der Harnröhre sich aufhalten. Und da wurden Provokationen durchgeführt mit fiebererzeugenden Mitteln. Und das waren meistens Vakzinen, also Bakterien. Der Gertler hat von sterilisierter Milch auch geschrieben und ja. Ja, das Verrückteste, was ich noch, als ich anfing, standen da noch einige Ampullen rum. Das ist dann nachher nicht mehr genommen worden. Das war sterilisiertes Terpentinöl. Das war die sogenannte, ja, Betonbombe. Da war also, das gab ein Ödem und dann konnte man, wenn man die im Gesäß hatte, also drei Tage nicht mehr laufen. Also das war das Übelste, was aber dann nicht mehr angewendet wurde, zumindestens bei uns. Aber diese Provokationsmethoden waren sogar vorgeschrieben, um dann nach der Behandlung die Heilung feststellen zu können. "589 Ende der 1970er und dann in den 1980er Jahren wurde ausschließlich Penicillin gegeben und nach der Gabe des Penicillins eine Provokation durchgeführt, um zu schauen, ob die Therapie erfolgreich war. Provokationen dienten demnach nicht mehr als eine mögliche Therapieform, sondern ausschließlich als Nachweis des Heilungserfolgs. Dafür wurden nicht mehr täglich Abstriche genommen, sondern „nach zwei, drei Kontrollabstrichen, wenn die negativ waren, [war] die Sache abgeschlossen und dabei war ein Abstrich nach einer Provokation“. Entsprechend war die Dauer des stationären Aufenthalts auf zwei, maximal drei Wochen beschränkt: „Ja, also wenn wir provoziert haben, Abstrich gemacht haben, und dann ohne Provokation der nächste Abstrich. (...) Und der übernächste auch unter Provokation, also drei Abstriche da negativ waren, war die Sache in der Regel erledigt. (...) Das kann eine Woche gedauert haben, kann aber auch kürzer gewesen sein. "590

Nicht zuletzt war der Wandel der Station 9 von einer geschlossenen zu einer offenen Station auch deshalb möglich, weil bestimmte Patientinnen nach Halle (Saale) überwiesen wurden. Die vom Führungsoffizier des IM Schneider beschriebene Methode der Überweisung von schwierigen Fällen auf die geschlossene Venerologische Station in Halle (Saale) wurde auch in Dresden praktiziert (Kap. 4.8): „Also wenn Sie jetzt so fragen, ich kann mich oder glaube mich erinnern zu können, dass wir irgendwann mal einen Fall nach Halle überwiesen haben. (...) Aber das wurde nicht von uns veranlasst. Das ging dann über den Kreisarzt, nicht also diese. Solche Maßnahmen waren geregelt. " ${ }^{591}$ Wer solche Ein- bzw. Überweisungen genau regelte, beispielsweise ein Gremium aus mehreren Personen, ausschließlich die Kreisärzte oder doch nur die Bezirksvenerologen, bleibt letztlich offen. Sicher ist, dass solche Überweisungen von Dresden aus praktiziert und die entsprechenden Patientinnen in der geschlossenen Venerologischen Station von Halle (Saale) aufgenommen wurden.

589 Interview mit Herrn DAA (Anm. 307).

590 Interview mit Herrn DAA (Anm. 307).

591 Interview mit Herrn DAA (Anm. 307). 